\title{
Simulations of radio emission from air showers with CORSIKA 8
}

\section{Nikolaos Karastathis, ${ }^{a, *}$ Remy Prechelt, ${ }^{b}$ Tim Huege ${ }^{a, d}$ and Juan Ammerman-Yebra ${ }^{c}$ on behalf of the CORSIKA 8 Collaboration}

(a complete list of authors can be found at the end of the proceedings)

${ }^{a}$ Institute for Astroparticle Physics (IAP), Karlsruhe Institute of Technology, Karlsruhe, Germany

${ }^{b}$ Department of Physics and Astronomy, University of Hawai'i Mānoa, Honolulu, USA

${ }^{c}$ Instituto Galego de Física de Altas Enerxías (IGFAE), Universidade de Santiago de Compostela, 15782

Santiago de Compostela, Spain

${ }^{d}$ Astrophysical Institute, Vrije Universiteit Brussel, Brussels, Belgium

E-mail: nikolaos.karastathis@kit.edu

CORSIKA 8 is a new framework for air shower simulations implemented in modern $\mathrm{C}++17$, based on past experience with existing codes like CORSIKA 7. The flexibility of this framework allows for the inclusion of radio-emission calculations as an integral part of the program. Our design makes radio simulations general and gives the user the freedom to choose between different formalisms, such as the "Endpoints" and "ZHS" formalisms. In addition, it takes advantage of the flexibility of the CORSIKA 8 environment and geometry design, allowing future updates to more complex scenarios such as showers crossing from air into dense media. We present first results, along with comparisons with other simulation programs like CoREAS in CORSIKA 7 and ZHAireS. In the future, based on our design, the opportunity arises for radio simulations to achieve a significant boost in performance by deploying parallel computing techniques, in particular employing GPUs, and hence, perform more sophisticated radio-emission studies.

$37^{\text {th }}$ International Cosmic Ray Conference (ICRC 2021)

July 12th - 23rd, 2021

Online - Berlin, Germany

\footnotetext{
*Presenter
} 


\section{Introduction}

Radio detection of extensive air showers has matured and become a technique competitive with "classical" particle and fluorescence detection over the past 20 years [1]. Due to the complexity of extensive air showers (EAS), detailed particle-level simulations of the radio emission of EAS are needed to analyze experimental data and reconstruct the properties of the primary particle. The two standard simulation tools used for simulations of the radio emission from showers are CoREAS [2] (implemented in CORSIKA 7 [3]) and ZHAireS [8]. These tools implement two different formalisms for calculating the radio emission from the particle tracks in the extensive air shower, namely the "Endpoint" formalism [4, 5] and the "ZHS" [7] formalism, respectively. However, these implementations inherit the limitations of their underlying shower codes and are not able to simulate the diverse array of current and future experiments (see section 1.1). Furthermore, as proposed next-generation experiments are growing significantly in size and channel-count, the computational cost of calculating the radio emission (especially for ultra high-energy showers) becomes intractable. To address these limitations, we have implemented the first radio emission module for the CORSIKA 8 (C8) shower simulation framework that is designed to be highly configurable and user-extensible to directly address the limitations of the current simulation tools and support the next generation of radio experiments.

\subsection{Limitations of existing air shower simulation codes}

The current generation of air shower simulation tools, CORSIKA 7 (C7) and ZHAireS, have several fundamental limitations that prevent their application to current and next-generation radio cosmic-ray and neutrino experiments. In particular,

Fixed down-going event geometry Both ZHAireS and C7 assume a flat or curvilinear approximation to the Earth and assume that all extensive air showers propagate downwards to the ground. ${ }^{1}$ Many funded and proposed experiments are sensitive to upwards-going air showers induced by $\tau$-leptons stratospheric cosmic-rays that never intersect the ground, or cosmic rays that are seen in reflection off the Earth's surface, none of which can be simulated with the established simulation tools. Furthermore, the approximate treatment of the curvature of the Earth in existing tools can introduce significant errors for highly-inclined downgoing-showers.

Straight-ray approximation Both existing codes assume that the radio emission propagates in a straight line from the emitting particle track to the receiver (i.e. the antenna), ignoring the refraction (or diffraction) of the radio emission as it propagates through the (potentially) changing refractivity of propagation medium. For highly-inclined or upgoing shower geometries, or for showers in media with strong refractivity gradients (i.e. polar ice), the ray curvature can be significant.

Fixed shower media The existing tools typically also only work with pre-programmed atmosphere models and are not easily configurable for alternate media which is a major limitation for the current and proposed experiments that need to simulate cosmic-ray or neutrino-induced showers in ice, the lunar regolith and even first air and then a dense medium.

\footnotetext{
${ }^{1}$ This statement applies to the official release versions - modified versions do exist but are available upon request.
} 


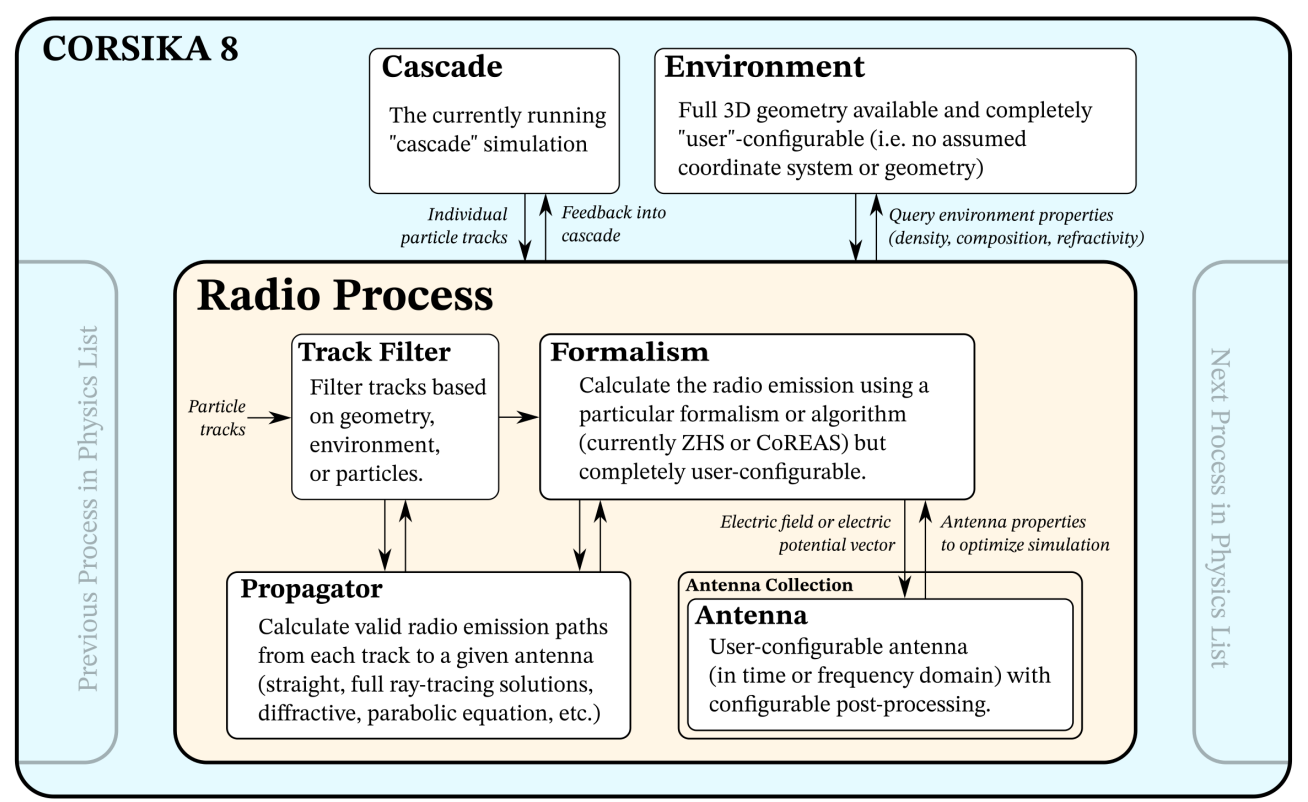

Figure 1: A schematic diagram of the radio process currently implemented in C8 and how it integrates with the $\mathrm{C} 8$ framework.

The design and implementation of the radio module in C8 has been tailored to address all of these limitations and provide a completely configurable and programmable platform to support the current and next generation of terrestrial and extra-terrestrial radio cosmic-ray and neutrino experiments.

\section{Architecture of Radio Emission Calculation in CORSIKA 8}

The radio module builds on C8's highly modular and extensible design to support the next generation of air shower experiments. The top-level architecture of the radio process is shown in Figure 1. Each component shown in Fig. 1 can be independently swapped out with CORSIKAprovided or user-provided $\mathrm{C}++$ code providing that they implement the required interfaces. The four primary configurable components are:

Filter The Filter is responsible for deciding which particles and tracks received from C8 should be forwarded to the radio emission calculation. This can be used to optimize the performance of the radio calculation by only processing certain particles (typically $e^{+} / e^{-}$), or by rejecting tracks that may not be visible (i.e. in a shadow region). It can also be used to "slice" simulations in terms of particle energy, atmospheric depth or other parameters.

Formalism The Formalism implements the specific radio emission calculation and orchestrates the core calculation. We have fully implemented two formalisms that calculate the electric field vector, namely the CoREAS algorithm [2] which implements the "Endpoints formalism" [4, 5] and the "ZHS" algorithm [7]. Both have been ported as closely as possible from their original 
implementations in the CoREAS [2] and ZHAireS [8] air shower simulation codes to allow for direct comparison before we investigate potential refinements.

Propagator The Propagator is used by the Formalism and Filter to calculate the (potentially multiple) valid radio signal propagation paths from each particle track to each antenna. We have already implemented two propagators that use a straight-ray approximation (akin to $\mathrm{C} 7$ and ZHAireS) including: 1) an analytic ray path solver that can only be used in media with uniform or exponential refractive indices; and 2) an integrating propagator that numerically integrates the time delay along each propagation path and can therefore work in arbitrarily complex media where no analytic solution exists. By separating the propagation of the radio signals from the emission formalism, C8 allows more advanced propagation techniques (e.g. full raytracing or parabolic equation methods) or more complex simulation scenarios (such as cross-media showers) to be implemented without requiring any modifications to the underlying emission formalisms.

Antenna The Antenna instance is storing, processing, and managing an individual antenna in the simulation. Multiple instances of independent antennas can be configured in the simulation and can apply their own unique processing to the received electric field, e.g., apply an antenna response. We currently implement a standard time-domain perfect antenna (frequency-domain can also be supported) that has a sample rate, start time, and a time window that is configurable individually for each antenna.

As our radio calculation is a standard process that is inserted into C8's process list, multiple instances of the radio emission can be simulated for the same shower. This immediately allows for comparing different radio implementations (e.g., comparing CoREAS against ZHS, or time-domain against frequency-domain implementations, etc.) on the exact same underlying shower, removing any uncertainty due to differences in the underlying shower or physics models [9]. In this work, we present the first radio pulses generated using $\mathrm{C} 8$ but also compare the CoREAS and ZHS algorithms (as implemented in C8) against reference pulses generated by the CoREAS formalism in C7 and the ZHS formalism in ZHAireS.

\section{Validation of the implementation}

\subsection{Electron in a uniform magnetic field}

We begin by validating the "CoREAS" and "ZHS" formalisms in C8 by calculating the synchrotron emission from a relativistic electron undergoing circular motion. Following the calculation in [4], we create a circular track of radius $L=100 \mathrm{~m}$ in the $x-y$ plane on which a $11.4 \mathrm{MeV}$ electron orbits in vacuum $(n=1)$ within a uniform magnetic field of 3.809 Gauss aligned with the $\mathrm{z}$-axis. The antenna is placed in the far field at a distance $R=30 \mathrm{~km}$ so that the unit vector from the point of emission to the antenna can be considered constant and lies along the $z$-axis.

We configure $\mathrm{C} 8$ using two different particle tracking (propagation) algorithms: 1) a simple algorithm that is used to connect 100,000 points that were explicitly constructed on a circular path with the proper radius with straight lines ("manual" tracking); and 2) the standard leapfrog integrator included in $\mathrm{C} 8$ to propagate particles within a magnetic field (" $\mathrm{C} 8$ tracking"). For the C8 tracking algorithm, we inject an electron with the correct energy and run the main C8 cascade 

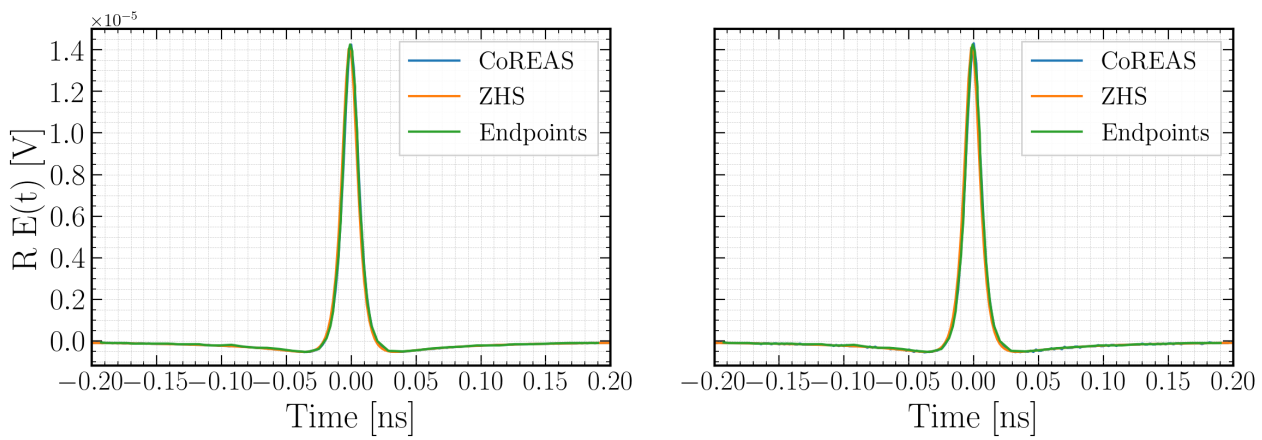

Figure 2: Radio pulse from an electron in a unifrom magnetic field performing a circular loop. "Manual tracking" algorithm (left) and "C8 tracking" (right). Both tracking algorithms are compared with the analytical solution of that system presented in [4] - Antenna is set at $R=30 \mathrm{~km}$.
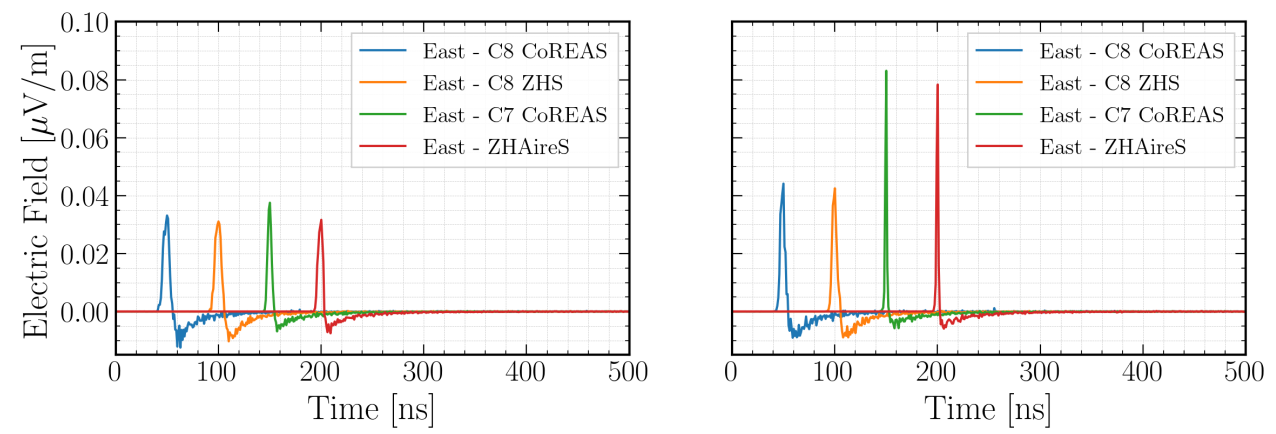

Figure 3: Signal pulse comparison for antenna at $50 \mathrm{~m}$ (left) and at $200 \mathrm{~m}$ (right) from the shower core. The East polarization of all pulses compared is shown where time offsets are arbitrary.

process for the (analytical) duration it would take for the electron to complete one orbit around its gyroradius. For each case, we simulate each shower with both "CoREAS" and "ZHS" formalisms simultaneously (each is otherwise configured identically). The synchrotron emission calculated by both formalisms is compared against the reference result from [4] in Fig. 2. Both agree extremely well with each other as well as the reference "Endpoints" pulse. We note that we need to configure the $\mathrm{C} 8$ tracking to perform very fine steps for accurate calculation of this extremely broad-band pulse.

We thus conclude that both the formalisms and the C 8 tracking (for very fine tracking) deliver correct results.

\subsection{Simulation of an extensive air shower}

We proceed to simulating the radio emission from full extensive air showers using C8. To keep complexity to a minimum, we restrict ourselves to simulating electron-induced showers. We use the "US Standard atmosphere", a uniform refractive index $(n=1.000327)$, and a constant horizontal geomagnetic field of $50 \mu \mathrm{T}$ aligned in the $x$ direction. We use the standard C8 electromagnetic interaction model ("PROPOSAL" [10]).

We use a $10 \mathrm{TeV}$ vertical shower as the baseline for these comparisons and use a star-shaped grid of antennas on 20 concentric rings spaced equally from $25 \mathrm{~m}$ to $500 \mathrm{~m}$ from the shower axis with 


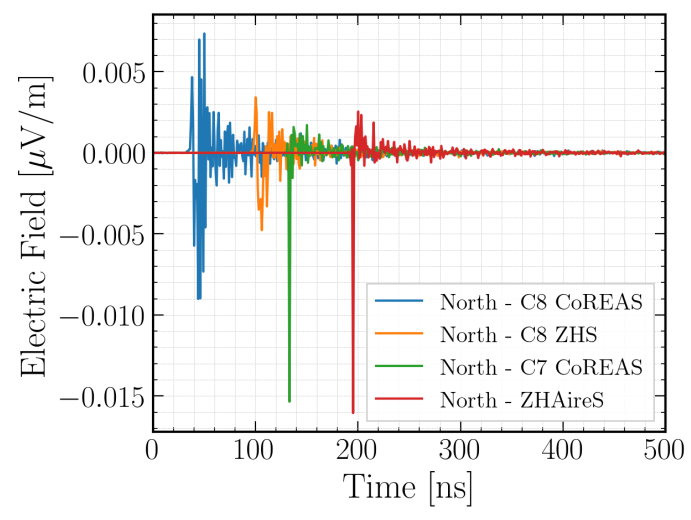

Figure 4: Signal pulse comparison for antenna at $200 \mathrm{~m}$ from the shower core - North polarization.

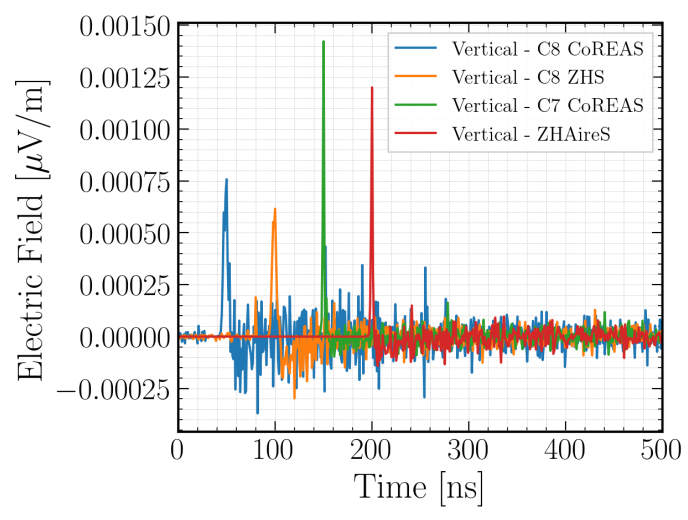

Figure 5: Signal pulse comparison for antenna at $200 \mathrm{~m}$ from the shower core - Vertical polarization.

8 antennas distributed azimuthally in each ring (see Fig. 7 for exact antenna locations). The depth of shower maximum of our simulation is $X_{\max } \approx 430 \mathrm{~g} \mathrm{~cm}^{-2}$. We note that particle energy cuts for electrons and positrons have been set to a comparably high value of $5 \mathrm{MeV}$ because of current limitations in the PROPOSAL version available in the radio branch of $\mathrm{C} 8$. Also, the tracking has been specifically configured in $\mathrm{C} 8$ to reduce the tracking step size (maximum deviation in the magnetic field of 0.0001 radians) as otherwise the resulting signal traces exhibited high-frequency noise which might point to an issue in the tracking that we still need to investigate in detail.

In Fig. 3 the East polarization of radio pulses calculated for two antennas at $50 \mathrm{~m}$ and at $200 \mathrm{~m}$ north of the shower axis with the C8-CoREAS and C8-ZHS implementations and compare it with pulses from a similar air shower (similar depth of maximum and number of particles at maximum) simulated with $\mathrm{C} 7$ and ZHAireS. The amplitudes for the antennas at $50 \mathrm{~m}$ (Fig. 3 left) agree within $12-15 \%$ between the $\mathrm{C} 8, \mathrm{C} 7$ and ZHAireS implementations. For antennas at $200 \mathrm{~m}$ from the shower axis (Fig. 3 right), there is a 50\% reduction in signal amplitude in $\mathrm{C} 8$ compared to $\mathrm{C} 7$ and ZHAireS and as well as a $100 \%$ increase in the pulse duration. The C 8 pulses are approximately $35 \%$ wider for antennas at $50 \mathrm{~m}$ and $200 \mathrm{~m}$. The polarization characteristics match very well between the $\mathrm{C} 8$ implementations and C7 as well as ZHAireS. This is evident for each polarization individually for the antennas at $200 \mathrm{~m}$ in Fig. 3 right (East), Fig. 4 (North) and Fig. 5 (Vertical). There is also very good agreement between C8-CoREAS and C8-ZHS, where the ZHS version exhibits slightly less noise on the pulse.

We also show the frequency spectra for two antennas located inside the Cherenkov ring (50 m and $200 \mathrm{~m}$ from the shower axis in Fig. 6). Both formalisms in C8 show an increase in power below $\sim 50 \mathrm{MHz}$ as compared with $\mathrm{C} 7$ and ZHaireS. It is evident that in C8 we are seeing less power at high frequencies with respect to $\mathrm{C} 7$ and ZHAireS which might indicate a small issue in time tracking in $\mathrm{C} 8$ or the electromagnetic model used; at the same time CoREAS and ZHS are close.

As a final comparison, we calculate and plot 2D maps of the energy fluence in the $30-80 \mathrm{MHz}$ band for both $\mathrm{C} 8$ formalisms as well as $\mathrm{C} 7$ and ZHAireS in Fig. 7. The absolute scale and polarization characteristics of all results agree qualitatively well, although slight differences become apparent, in particular an offset from the symmetry axis in the $\vec{v} \times(\vec{v} \times \vec{B})$ polarization in the C8 and ZHAireS results which is not present in C7 and a dot in the $\vec{v}$ polarization for only C8 ZHS and ZHAireS. 

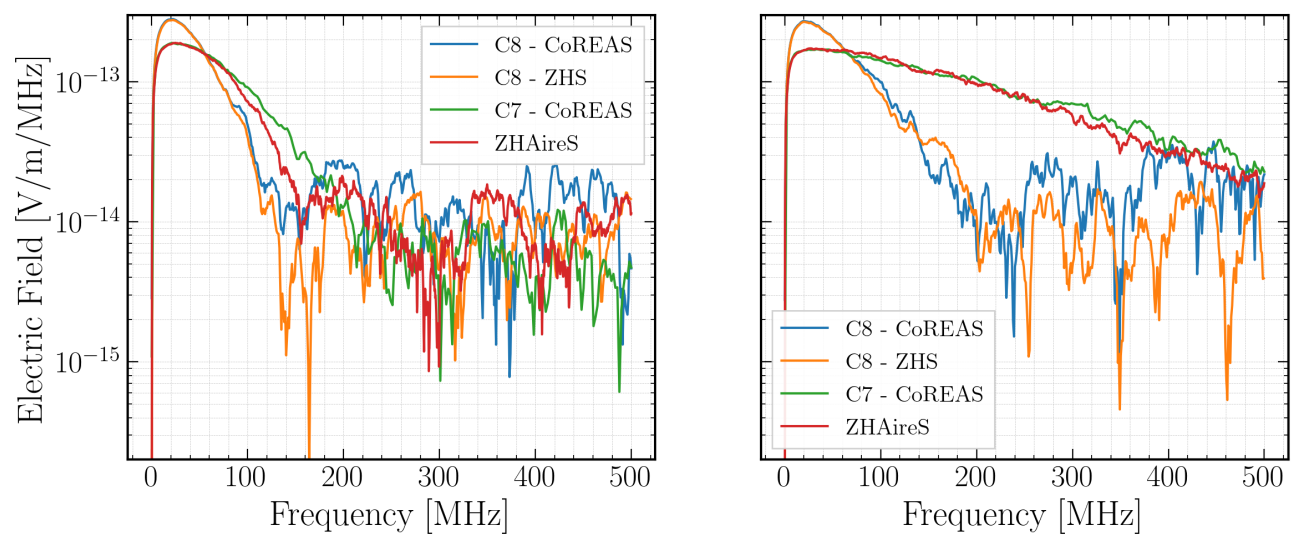

Figure 6: Frequency spectra for antenna at $50 \mathrm{~m}$ (left) and at $200 \mathrm{~m}$ (right) from the shower core.

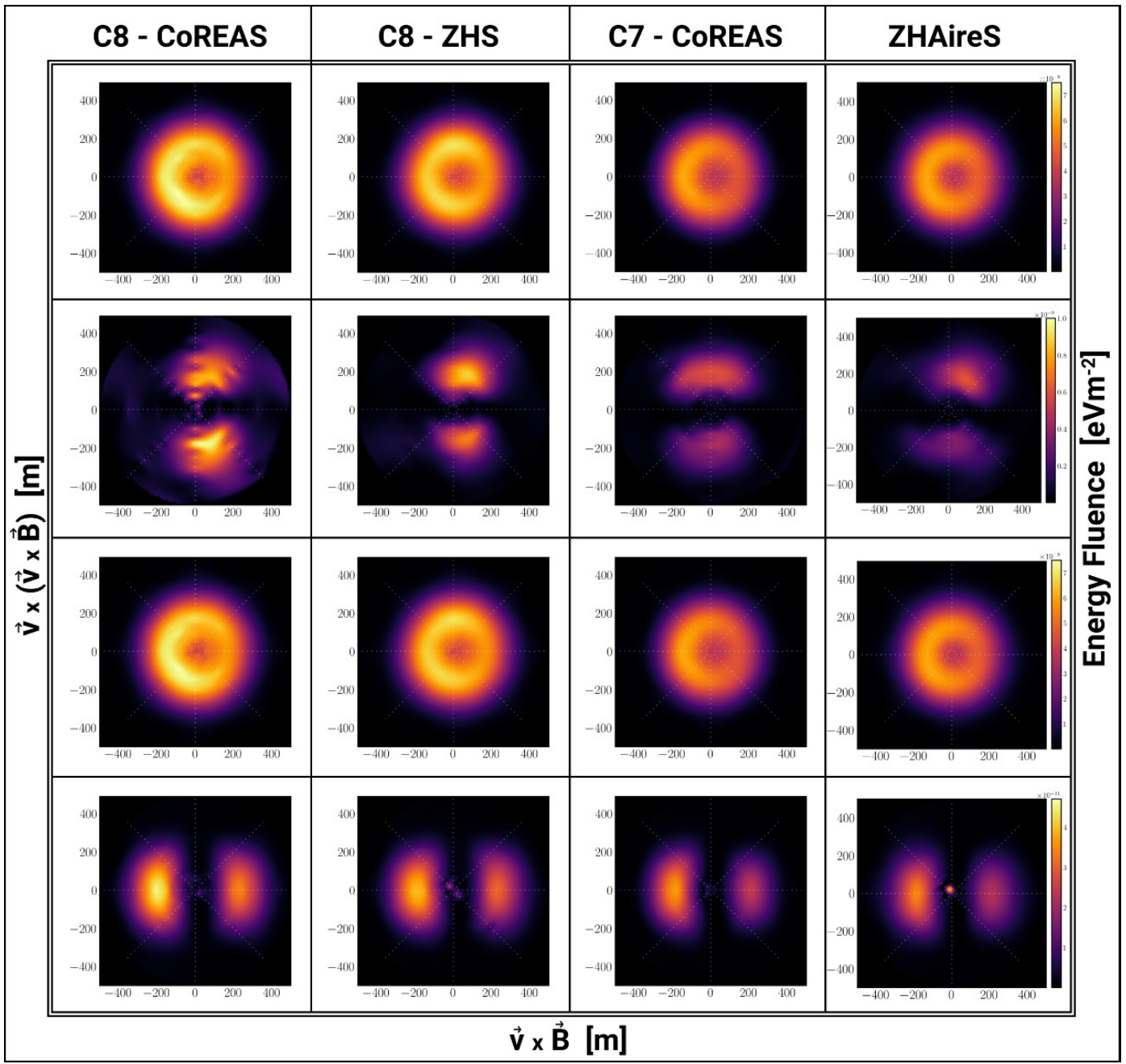

Figure 7: Table of energy fluence in different polarizations of the electric field for C8 CoREAS, C8 ZHS, C7 CoREAS and ZHAireS. The order of the polarizations we see starting from top to bottom is: all polarizations, $\vec{v} \times(\vec{v} \times \vec{B}), \vec{v} \times \vec{B}$ and $\vec{v}$. 


\section{Conclusions}

We have completed a first implementation of radio-emission calculations in C8 based on exact reproductions of the CoREAS and ZHS algorithms implemented in a modern, modular structure that will allow flexible extension for the needs of current and future experiments.

We have performed low-level tests with single electrons undergoing circular motion to establish that the radio-emission calculation as well as the particle tracking in the magnetic field in $\mathrm{C} 8$ work correctly. Afterwards, we have performed simulations of a vertical $10 \mathrm{TeV}$ electron shower in a uniform refractive index and compared the results between a reference simulation with $\mathrm{C} 7$ as well as ZHAireS and our implementation of the CoREAS and ZHS algorithms in C8. We observe an agreement at the level of better than a factor of two in absolute amplitude, a good agreement of polarization characteristics and a very good agreement between the CoREAS and ZHS implementations in C8. Notably, the radio pulses simulated with C8 are wider and have less high-frequency content.

We note that the air-shower results depend on many ingredients, in particular the underlying electromagnetic interaction model PROPOSAL and the tracking in the magnetic field (which likely needs improvements as we needed to track much finer in $\mathrm{C} 8$ than in $\mathrm{C} 7$ for instance to achieve comparably clean radio pulses). Having reached this major milestone, we will address the observed differences by detailed studies in the future.

\section{References}

[1] T. Huege, Phys. Rept. 620 (2016), 1-52 [1601.07426].

[2] T. Huege, M. Ludwig and C. W. James, AIP Conf. Proc. 1535 (2013) 1, 128 [1301.2132].

[3] D. Heck, J. Knapp, J.N. Capdevielle, G. Schatz, and T. Thouw, FZKA Report 6019, 1998.

[4] T. Huege, C. W. James, H. Falcke and M. Ludwig, Proc. 32nd Int. Cosmic Ray Conf. (2011) 4, 308 [1112.2126]

[5] M. Ludwig and T. Huege, Astropart. Phys. 34 (2011), 438-446 [1010.5343]

[6] R. Engel, D. Heck, T. Huege, T. Pierog, M. Reininghaus, F. Riehn, R. Ulrich, M. Unger and D. Veberič, Comput. Softw. Big Sci. 3 (2019) 1, 2 [1808.08226].

[7] E. Zas, F. Halzen and T. Stanev, Phys. Rev. D 45 (1992), 362-376

[8] J. Alvarez-Muñiz, W. R. Carvalho, Jr., M. Tueros and E. Zas, Astropart. Phys. 35 (2012), 287-299 [1005.0552]

[9] M. Gottowik, C. Glaser, T. Huege and J. Rautenberg, Astropart. Phys. 103 (2018), 87-93 [1712.07442].

[10] J. M. Alameddine, J. Soedingrekso, A. Sandrock, M. Sackel and W. Rhode, J. Phys. Conf. Ser. 1690 (2020) 1, 012021 


\section{Full Authors List: CORSIKA 8 Collaboration}

Jean-Marco Alameddine ${ }^{b}$, Johannes Albrecht ${ }^{b}$, Jaime Alvarez-Muñiz ${ }^{r}$, Antonio Augusto Alves $\mathrm{Jr}^{d}$, Luisa Arrabito ${ }^{a}$, Dominik Baack ${ }^{b}$, Konrad Bernlöhr ${ }^{c}$, Marcus Bleicher ${ }^{o}$, Johan Bregeon ${ }^{s}$, Mathieu Carrere ${ }^{a}$, Hans Dembinski ${ }^{b}$, Hannah Elfner ${ }^{i}$, Dominik Elsässer ${ }^{b}$, Ralph Engel ${ }^{d}$, Hu Fan ${ }^{p}$, Anatoli Fedynitch ${ }^{j}$, Dieter Heck ${ }^{d}$, Tim Huege ${ }^{d, e}$, Karl-Heinz Kampert ${ }^{k}$, Nikolaos Karastathis ${ }^{d}$, Lukas Nellen ${ }^{f}$, Maximilian Nöthe ${ }^{b}$, David Parello ${ }^{t}$, Tanguy Pierog ${ }^{d}$, Maria Pokrandt ${ }^{d}$, Anton Poctarev ${ }^{d}$, Remy Prechelt ${ }^{l}$, Maximilian Reininghaus $^{d, m}$, Wolfgang Rhode ${ }^{b}$, Felix Riehn ${ }^{h, r}$, Maximilian Sackel ${ }^{b}$, Alexander Sandrock ${ }^{u}$, Pranav Sampathkumar ${ }^{d}$, Michael Schmelling $^{c}$, André Schmidt ${ }^{d}$, Günter Sigl ${ }^{n}$, Jan Soedingrekso ${ }^{b}$, Bernhard $\operatorname{Spaan}^{b}$, Donglian $\mathrm{Xu}^{q}$, Juan Ammerman-Yebra ${ }^{r}$ and Enrique $\mathrm{Zas}^{r}$

${ }^{a}$ Laboratoire Univers et Particules, Université de Montpellier 2, Montpellier, France, ${ }^{b}$ Experimentelle Physik 5, TU Dortmund, Dortmund, Germany, ${ }^{c}$ Max Planck Institute for Nuclear Physics, Heidelberg, Germany, ${ }^{d}$ Institute for Astroparticle Physics, Karlsruhe Institute of Technology, Karlsruhe, Germany, ${ }^{e}$ Astrophysical Institute, Vrije Universiteit Brussel, Brussels, Belgium ${ }^{f}$ National Autonomous University of Mexico, Mexico City, Mexico, ${ }^{g}$ Instituto de Tecnologías en Detección y Astropartículas, Buenos Aires, Argentina, ${ }^{h}$ Laboratory of Instrumentation and Experimental Particles, Lisbon, Portugal, ${ }^{i}$ Helmholtzzentrum für Schwerionenforschung, Darmstadt, Germany, ${ }^{j}$ Institute for Cosmic Ray Research, The University of Tokyo, Tokyo, Japan, ${ }^{k}$ Lehrstuhl für Astroteilchenphysik, Bergische Universität Wuppertal, Wuppertal, Germany, ${ }^{l}$ Department of Physics \& Astronomy, University of Hawai'i at Manoa, Honolulu, USA, ${ }^{m}$ Instituto de Tecnologías en Detección y Astropartículas, Buenos Aires, Argentina, ${ }^{n}$ II Institut für Theoretische Physik, Universität Hamburg, Hamburg, Germany, ${ }^{o}$ Johann-Wolfgang-Goethe-Universitaet, Frankfurt am Main, Germany, ${ }^{p}$ Peking University, Beijing, China, ${ }^{q}$ Tsung-Dao Lee Institute, Shanghai, China, ${ }^{r}$ Instituto Galego de Física de Altas Enerxías, Universidade de Santiago de Compostela, Santiago de Compostela, Spain, ${ }^{s}$ Laboratoire de Physique Subatomique et de Cosmologie, Grenoble, France, ${ }^{t}$ LIRMM, Univ Montpellier, CNRS, Montpellier, France, ${ }^{u}$ National Research Nuclear University, Moscow Engineering Physics Institute, Moscow, Russia.

\section{Acknowledgements}

For the simulations presented, computing resources from KIT have been used. This work has also received financial support from Xunta de Galicia (Centro singular de investigación de Galicia accreditation 2019-2022), by European Union ERDF, by the "María de Maeztu" Units of Excellence program MDM-2016-0692, the Spanish Research State Agency and from Ministerio de Ciencia e Innovación PID2019-105544GB-I00 and RED2018-102661-T (RENATA). 